Network Working Group

Request for Comments: 3433

Category: Standards Track
A. Bierman Cisco Systems, Inc.

D. Romascanu

Avaya Inc.

K.C. Norseth

L-3 Communications

December 2002

\title{
Entity Sensor Management Information Base
}

\section{Status of this Memo}

This document specifies an Internet standards track protocol for the Internet community, and requests discussion and suggestions for improvements. Please refer to the current edition of the "Internet Official Protocol Standards" (STD 1) for the standardization state and status of this protocol. Distribution of this memo is unlimited.

Copyright Notice

Copyright (C) The Internet Society (2002). All Rights Reserved.

Abstract

This memo defines a portion of the Management Information Base (MIB) for use with network management protocols in the Internet community. In particular, it describes managed objects for extending the Entity MIB (RFC 2737) to provide generalized access to information related to physical sensors, which are often found in networking equipment (such as chassis temperature, fan RPM, power supply voltage).

Table of Contents

1 The Internet-Standard Management Framework ............ 2

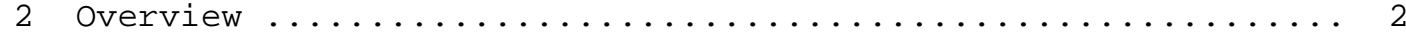

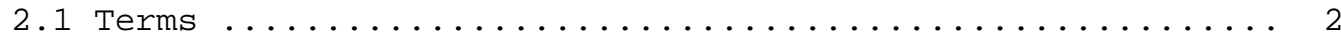

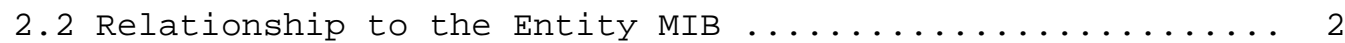

2.3 Relationship to General Thresholding Mechanisms ....... 3

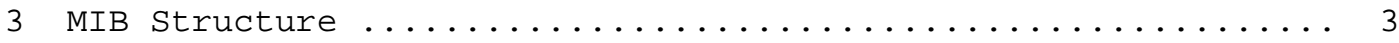

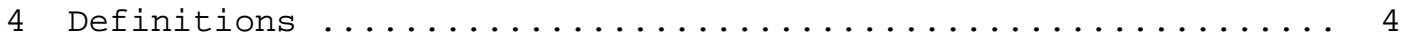

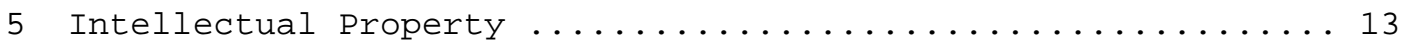

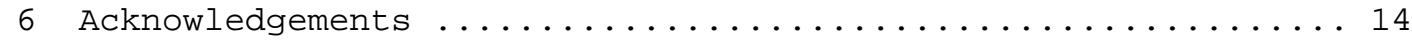

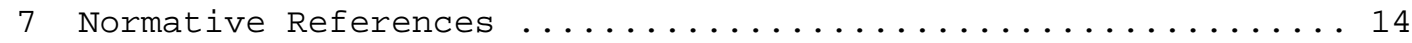

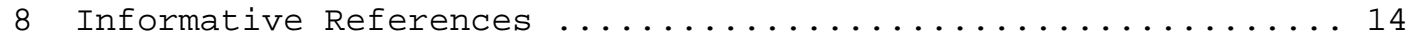

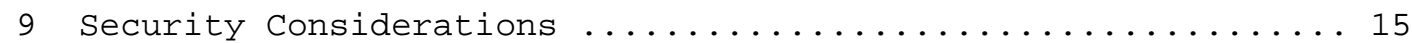

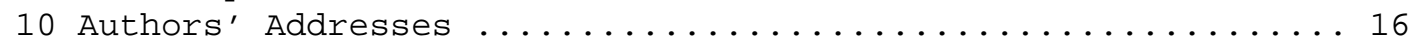

11 Full Copyright Statement $\ldots \ldots \ldots \ldots \ldots \ldots \ldots \ldots \ldots \ldots \ldots \ldots \ldots \ldots \ldots \ldots$ 
1. The Internet-Standard Management Framework

For a detailed overview of the documents that describe the current Internet-Standard Management Framework, please refer to section 7 of RFC 3410 [RFC3410].

Managed objects are accessed via a virtual information store, termed the Management Information Base or MIB. MIB objects are generally accessed through the Simple Network Management Protocol (SNMP). Objects in the MIB are defined using the mechanisms defined in the Structure of Management Information (SMI). This memo specifies a MIB module that is compliant to the SMIV2, which is described in STD 58, RFC 2578 [RFC2578], STD 58, RFC 2579 [RFC2579] and STD 58, RFC 2580 $[\mathrm{RFC} 2580]$.

\section{Overview}

There is a need for a standardized way of obtaining information related to the physical sensors which are commonly found in networking equipment. Information such as the current value of the sensor, the current operational status, and the data units precision associated with the sensor, should be represented in a consistent manner for any type of sensor.

Physical sensors are represented in the Entity MIB with entPhysicalEntry and an entPhysicalclass value of 'sensor(8)'. The information provided in the ENTITY-SENSOR-MIB module (defined in this document) defines a sparse augmentation of the entPhysicaltable, for entries which represent physical sensors.

\subsection{Terms}

The key words "MUST", "MUST NOT", "REQUIRED", "SHALL", "SHALL NOT", "SHOULD", "SHOULD NOT", "RECOMMENDED", "MAY", and "OPTIONAL" in this document are to be interpreted as described in BCP 14, RFC 2119. [RFC2119]

\subsection{Relationship to the Entity MIB}

The MIB objects defined in this document provide a sparse augmentation to the entPhysicalTable in the Entity MIB, for entries in which the associated entPhysicalclass object is equal to 'sensor(8)'. An agent is expected to maintain an entPhySensorEntry with the same entPhysicalindex value for each entPhysicalEntry representing a physical sensor. Therefore, implementation of the entityphysicalGroup is required for agents that implement the Entity Sensor MIB. 


\subsection{Relationship to General Thresholding Mechanisms}

There are no specialized sensor value thresholding mechanisms defined in this MIB module. Instead, it is recommended that a generalized thresholding MIB, such as the mechanisms defined by the Alarm and Events groups of the Remote Network Monitoring MIB [RFC2819], be used for this purpose.

\section{MIB structure}

The Entity Sensor MIB contains a single group called the entitysensorValueGroup, which allows objects to convey the current value and status of a physical sensor.

The entitySensorValuegroup contains a single table, called the entPhysensorTable, which provides a small number of read-only objects:

entPhySensorType

This object identifies the type of data units associated with the sensor value.

entPhySensorScale

This object identifies the (power of 10) scaling factor associated with the sensor value.

entPhySensorPrecision

This object identifies the number of decimal places of precision associated with the sensor value.

entPhySensorValue

This object identifies the current value of the sensor.

entPhySensorOperstatus

This object identifies the current operational status of the sensor (as it's known to the agent).

entPhySensorUnitsDisplay

This object provides a textual description of the data units represented by the entPhySensorType and entPhysensorscale objects.

entPhySensorValueTimestamp

The object identifies the value of sysupTime at the time the agent last updated the information in the entry. This object is only relevant if the agent uses a polling implementation strategy, (i.e., the associated entPhysensorValueUpdateRate object is greater than zerol. 


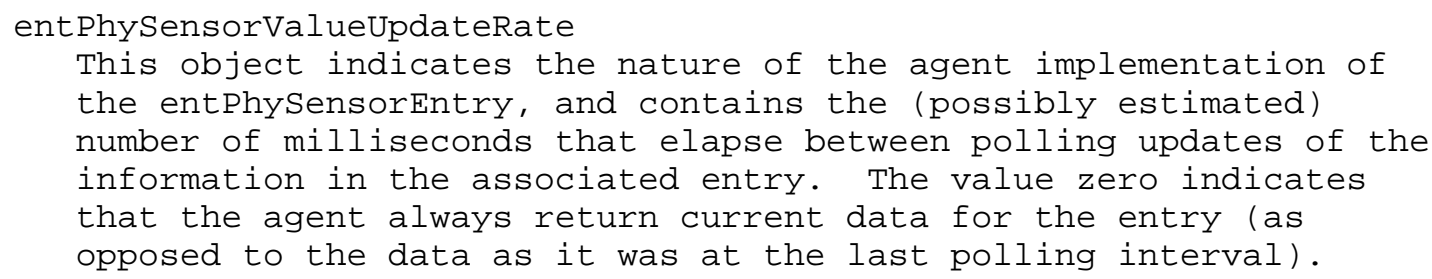

4. Definitions

ENTITY-SENSOR-MIB DEFINITIONS : := BEGIN

IMPORTS

MODULE-IDENTITY, OBJECT-TYPE,

Integer32, Unsigned32, mib-2

FROM SNMPV2-SMI

MODULE-COMPLIANCE, OBJECT-GROUP

FROM SNMPV2-CONF

TEXTUAL-CONVENTION, TimeStamp

FROM SNMPV2-TC

entPhysicalindex, entityPhysicalGroup

FROM ENTITY-MIB

SnmpAdminstring

FROM SNMP-FRAMEWORK-MIB;

entitySensorMIB MODULE-IDENTITY

LAST-UPDATED "200212160000Z"

ORGANIZATION "IETF Entity MIB Working Group"

CONTACT-INFO

"

Andy Bierman

Cisco Systems, Inc.

Tel: +1 408-527-3711

E-mail: abierman@cisco.com

Postal: 170 West Tasman Drive

San Jose, CA USA 95134

Dan Romascanu

Avaya Inc.

Tel: +972-3-645-8414

Email: dromascalavaya.com

Postal: Atidim technology Park, Bldg. \#3

Tel Aviv, Israel, 61131

K.C. Norseth

L-3 Communications

Tel: +1 801-594-2809

Email: kenyon.c.norseth@L-3com.com

Postal: 640 N. 2200 West.

Bierman, et. al.

Standards Track

[Page 4] 


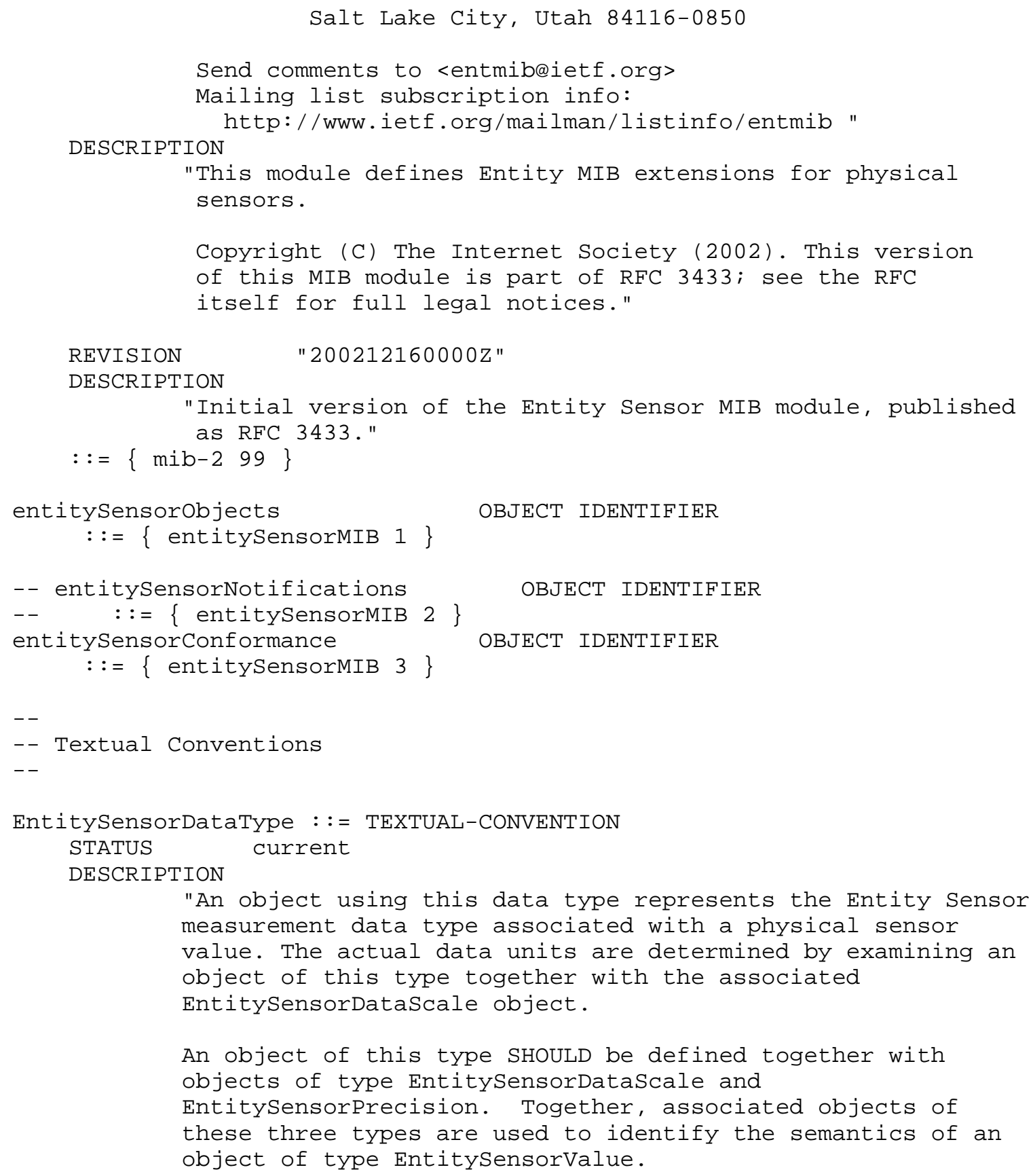




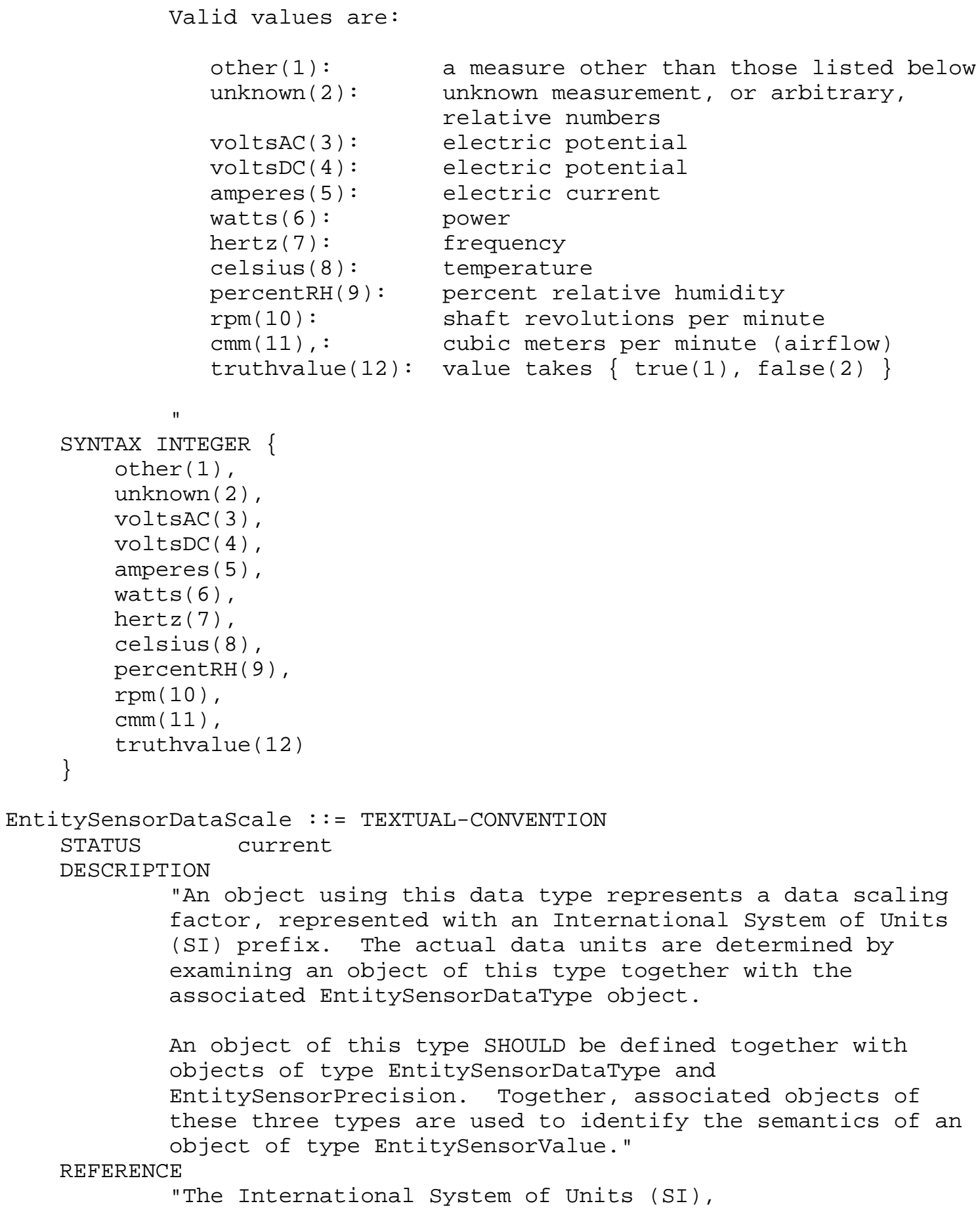




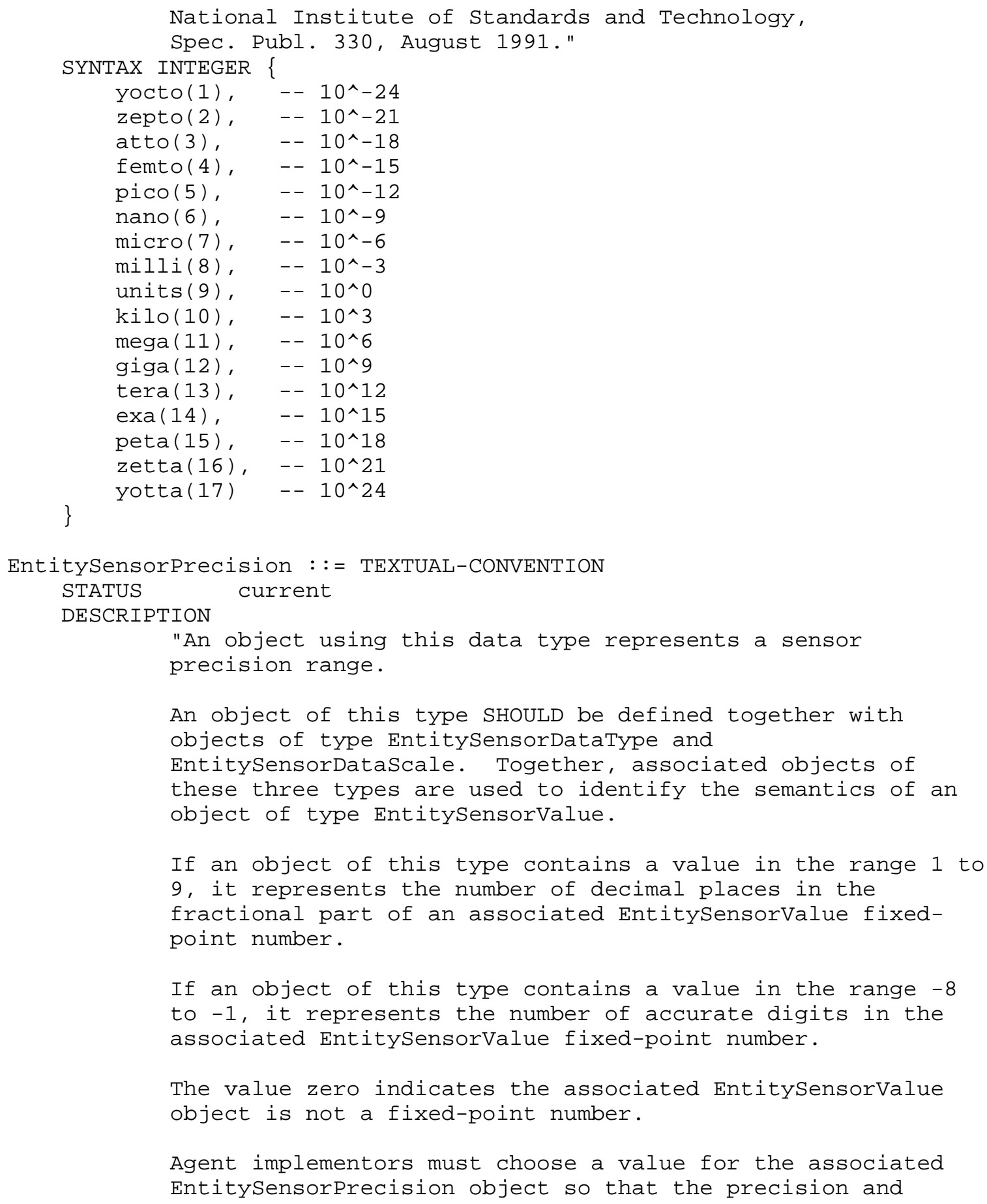


accuracy of the associated Entitysensorvalue object is correctly indicated.

For example, a physical entity representing a temperature sensor that can measure 0 degrees to 100 degrees $\mathrm{C}$ in 0.1 degree increments, +/- 0.05 degrees, would have an EntitysensorPrecision value of ' 1 ', an EntitysensorDatascale value of 'units(9)', and an EntitySensorvalue ranging from '0' to '1000'. The EntitySensorvalue would be interpreted as 'degrees $\mathrm{C}$ * $10^{\prime} . "$

SYNTAX Integer32 (-8..9)

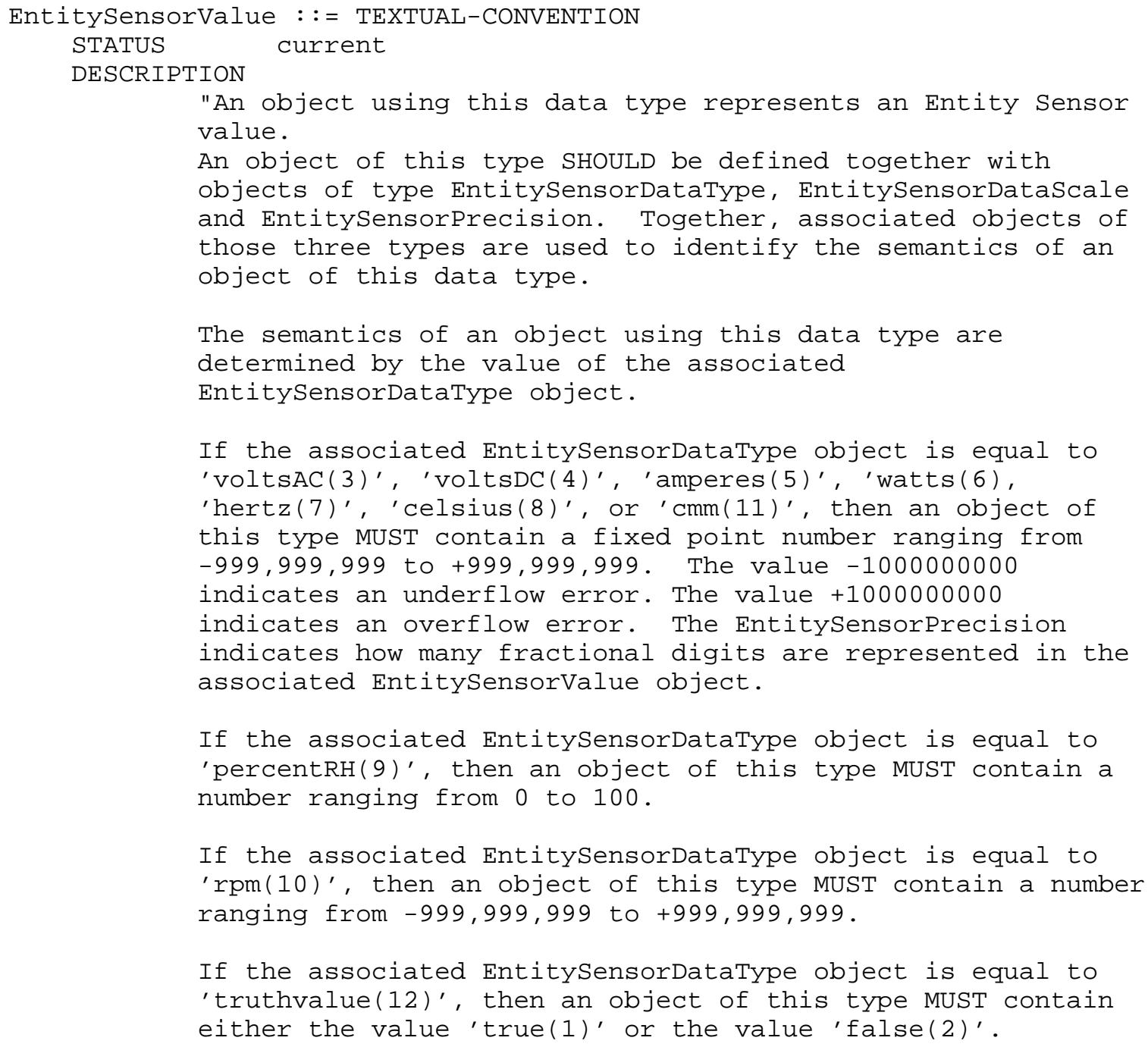




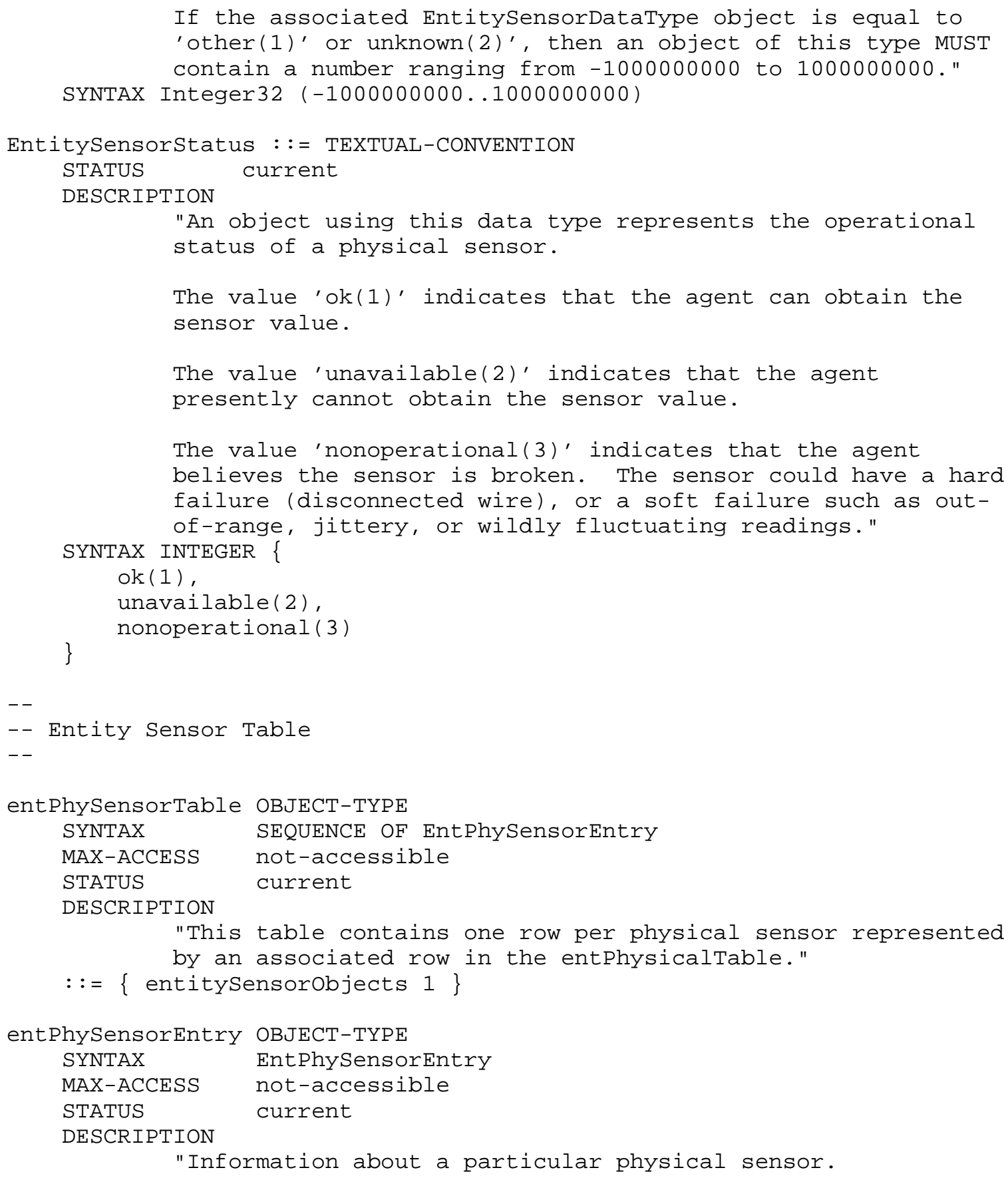




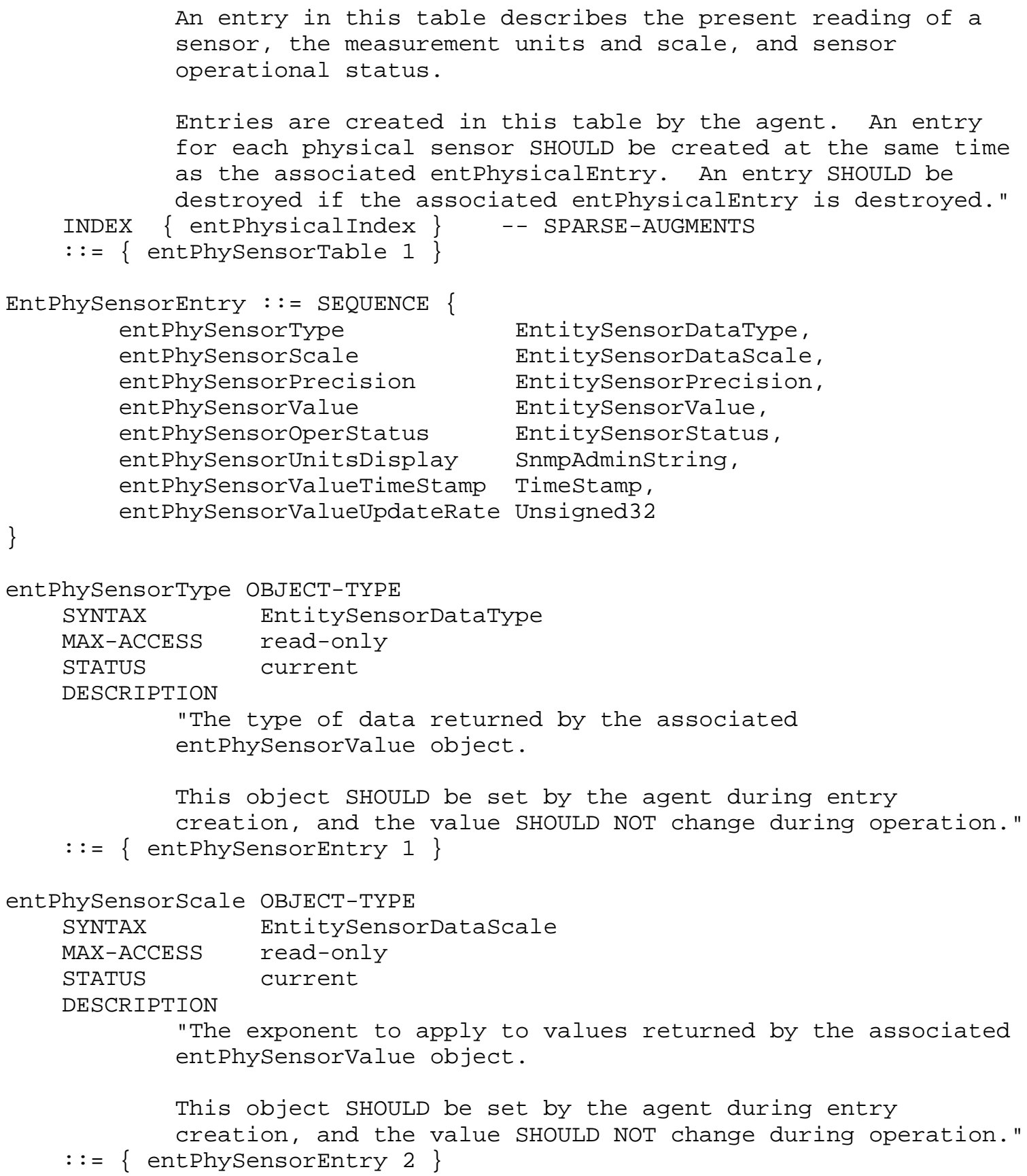

Bierman, et. al.

Standards Track

[Page 10] 


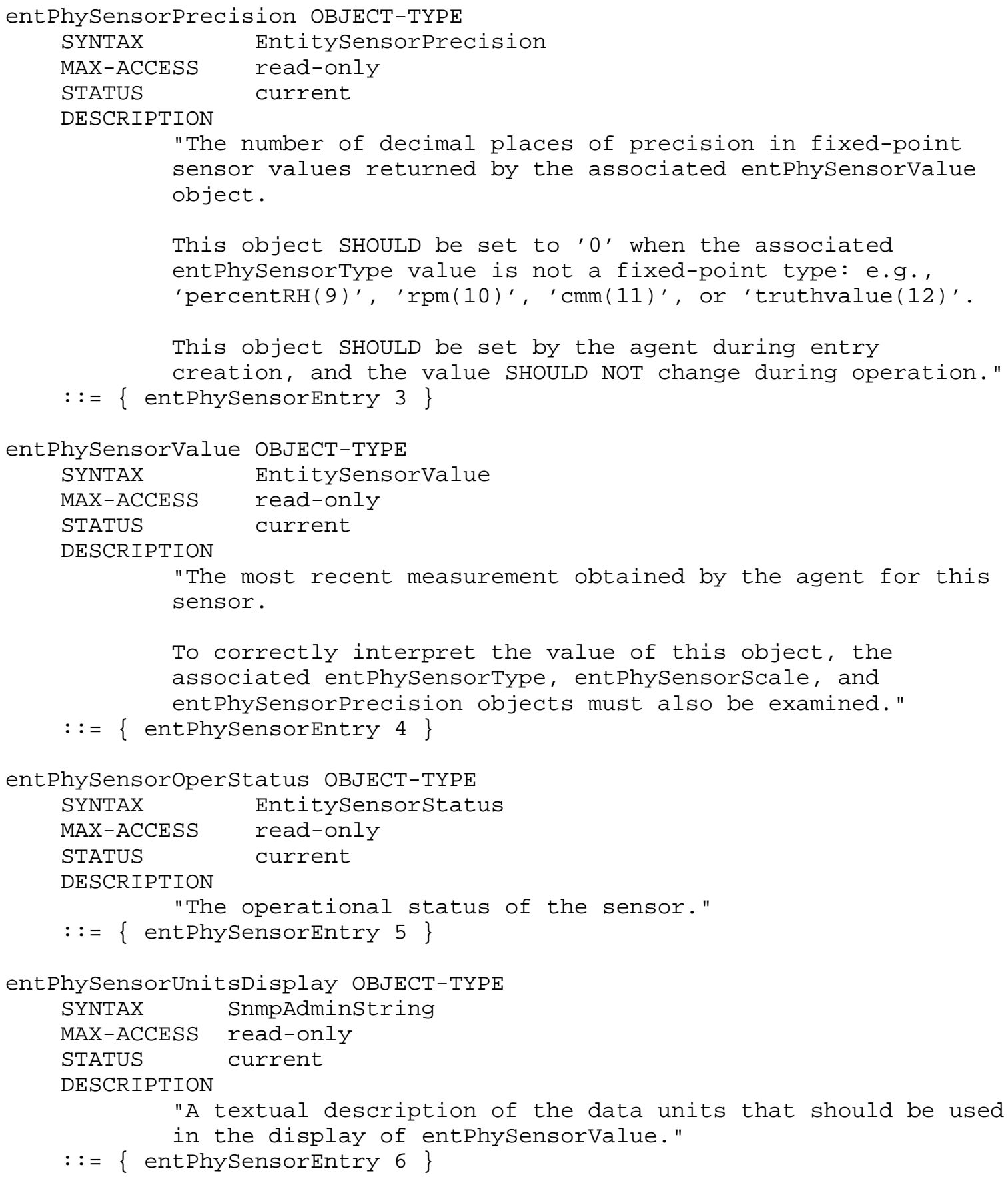

"A textual description of the data units that should be used in the display of entPhySensorvalue." 


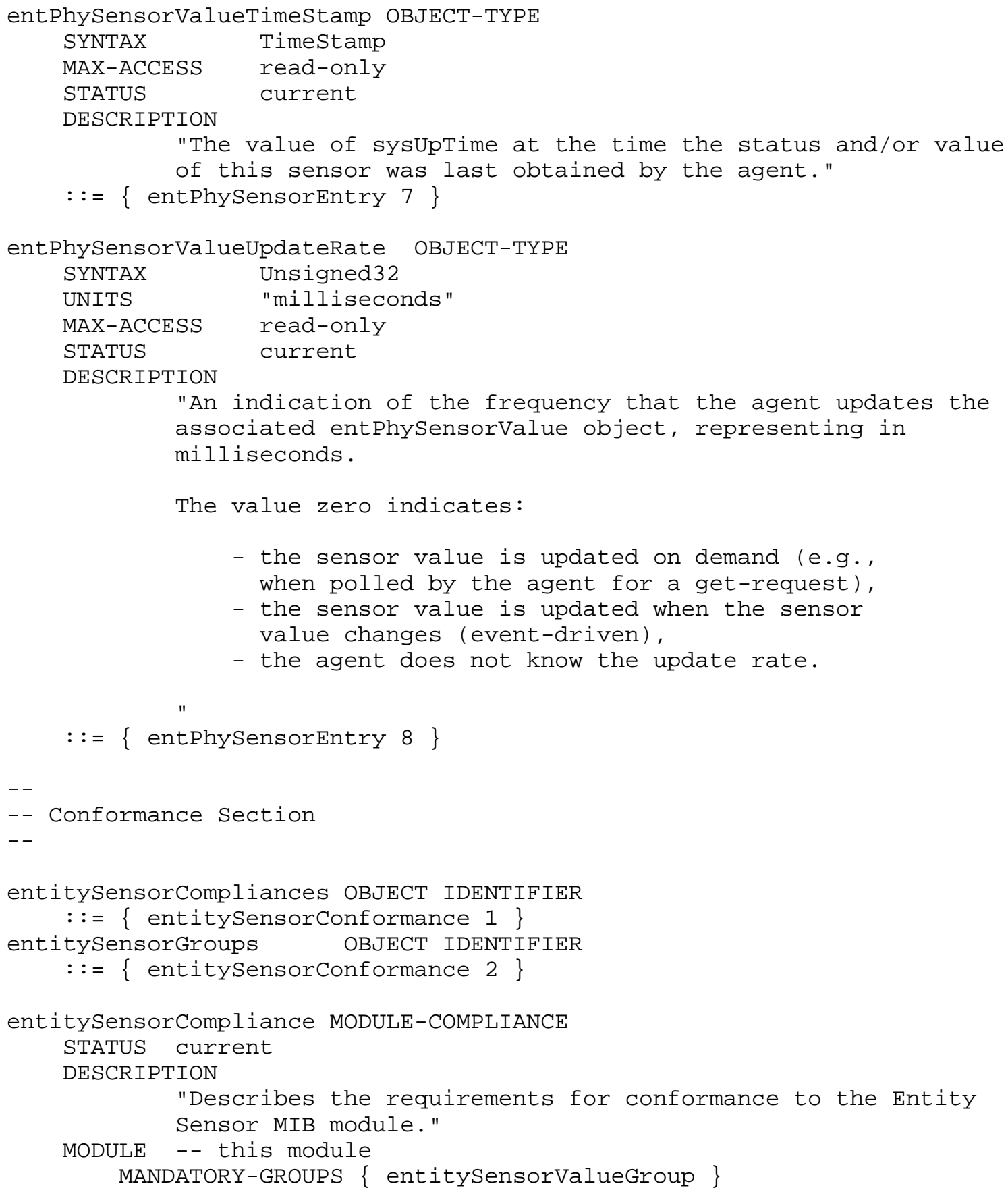




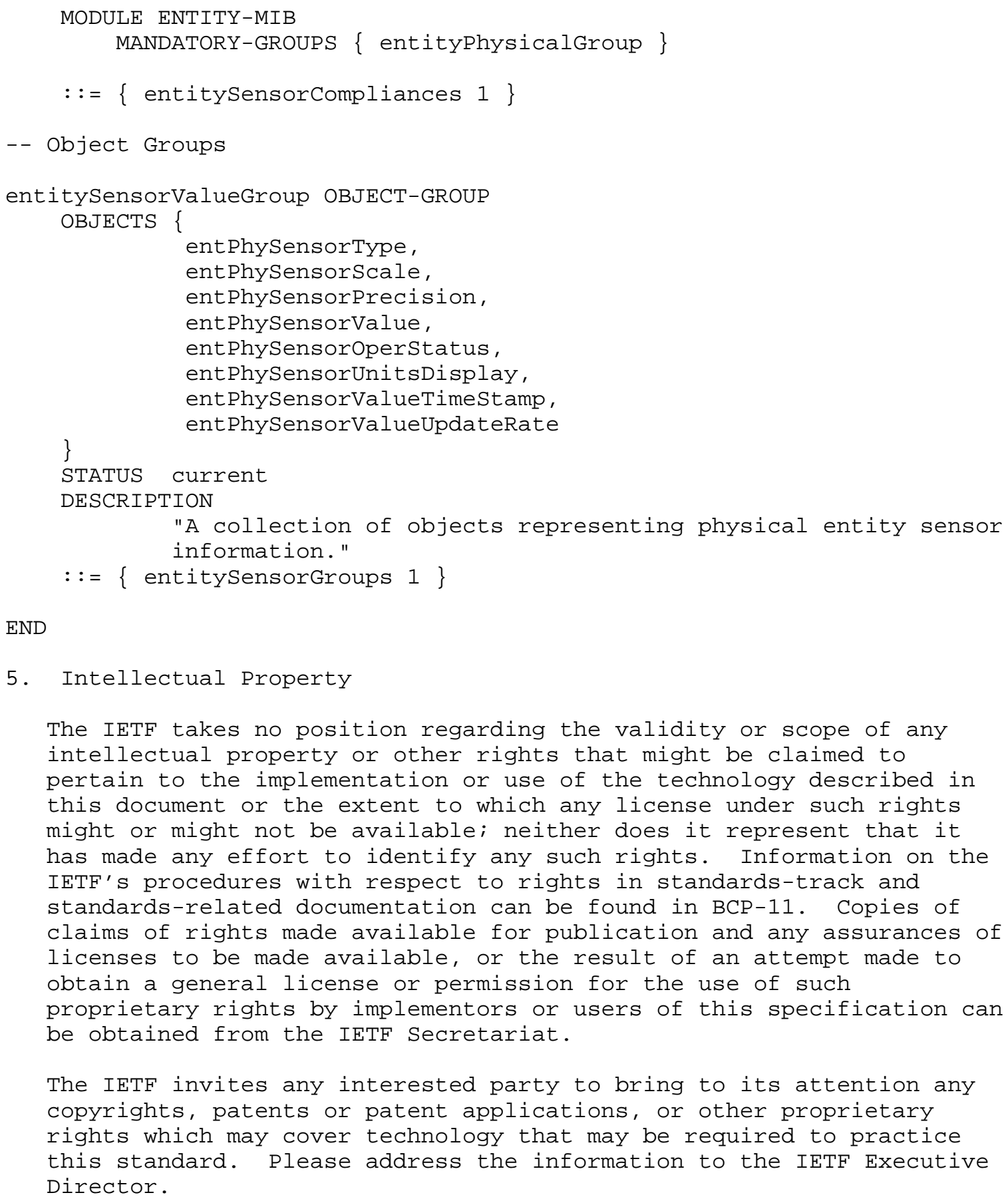




\section{Acknowledgements}

This memo is a product of the Entity MIB working group. It is based on an existing proprietary MIB module written by Cliff Sojourner.

7. Normative References

[RFC2119] Bradner, S., "Key words for use in RFCs to Indicate Requirement Levels", BCP 14, RFC 2119, March 1997.

[RFC2578] McCloghrie, K., Perkins, D., Schoenwaelder, J., Case, J., Rose, M. and S. Waldbusser, "Structure of Management Information Version 2 (SMIV2)", STD 58, RFC 2578, April 1999.

[RFC2579] McCloghrie, K., Perkins, D., Schoenwaelder, J., Case, J., Rose, M., and S. Waldbusser, "Textual Conventions for SMIv2", STD 58, RFC 2579, April 1999.

[RFC2580] McCloghrie, K., Perkins, D., Schoenwaelder, J., Case, J., Rose, M. and S. Waldbusser, "Conformance Statements for SMIv2", STD 58, RFC 2580, April 1999.

[RFC2737] McCloghrie, K. and A. Bierman, "Entity MIB (Version 2)", RFC 2737, December 1999.

[RFC3414] Blumenthal, U. and B. Wijnen, "User-based Security Model (USM) for version 3 of the Simple Network Management Protocol (SNMPV3)", STD 62, RFC 3414, December 2002.

[RFC3415] Wijnen, B., Presuhn, R. and K. McCloghrie, "View-based Access Control Model (VACM) for the Simple Network Management Protocol (SNMP)", STD 62, RFC 3415, December 2002 .

8. Informative References

[RFC2819] Waldbusser, S., "Remote network Monitoring Management Information Base", RFC 2819, May 2000.

[RFC3410] Case, J., Mundy, R., Partain, D. and B. Stewart, "Introduction and Applicability Statements for InternetStandard Management Framework", RFC 3410, December 2002. 
9. Security Considerations

There is one managed object in this MIB that may contain sensitive information. This is:

entPhySensorValue

This object may expose the values of particular physical sensors for a device.

SNMPVl by itself is not a secure environment. Even if the network itself is secure (for example by using IPSec), there is no control as to who on the secure network is allowed to access and GET/SET (read/change/create/delete) the objects in this MIB.

It is recommended that the implementors consider the security features as provided by the SNMPv3 framework. Specifically, the use of the User-based Security Model STD 62, RFC 3414 [RFC3414] and the View-based Access Control Model STD 62, RFC 3415 [RFC3415] is recommended.

It is then a customer/user responsibility to ensure that the SNMP entity giving access to an instance of this MIB, is properly configured to give access to only the objects, and those principals (users) that have legitimate rights to indeed GET or SET (change/create/delete) them. 
10. Authors' Addresses

Andy Bierman

Cisco Systems, Inc.

170 West Tasman Drive

San Jose, CA USA 95134

Phone: +1 408-527-3711

EMail: abiermandcisco.com

Dan Romascanu

Avaya Inc.

Atidim Technology Park, Bldg. \#3

Tel Aviv, 61131, Israel

Phone: +972-3-545-8414

EMail: dromascalavaya.com

K.C. Norseth

L-3 Communications

640 N. 2200 West.

Salt Lake City, Utah 84116-0850

Phone: +1 801-594-2809

EMail: kenyon.c.norseth@L-3com.com 
11. Full Copyright statement

Copyright (C) The Internet Society (2002). All Rights Reserved.

This document and translations of it may be copied and furnished to others, and derivative works that comment on or otherwise explain it or assist in its implementation may be prepared, copied, published and distributed, in whole or in part, without restriction of any kind, provided that the above copyright notice and this paragraph are included on all such copies and derivative works. However, this document itself may not be modified in any way, such as by removing the copyright notice or references to the Internet society or other Internet organizations, except as needed for the purpose of developing Internet standards in which case the procedures for copyrights defined in the Internet Standards process must be followed, or as required to translate it into languages other than English.

The limited permissions granted above are perpetual and will not be revoked by the Internet society or its successors or assigns.

This document and the information contained herein is provided on an "AS IS" basis and THE INTERNET SOCIETY AND THE INTERNET ENGINEERING TASK FORCE DISCLAIMS ALL WARRANTIES, EXPRESS OR IMPLIED, INCLUDING BUT NOT LIMITED TO ANY WARRANTY THAT THE USE OF THE INFORMATION HEREIN WILL NOT INFRINGE ANY RIGHTS OR ANY IMPLIED WARRANTIES OF MERCHANTABILITY OR FITNESS FOR A PARTICULAR PURPOSE.

Acknowledgement

Funding for the RFC Editor function is currently provided by the Internet society. 